\title{
Non-Universal Gaugino Masses, CDMS, and the LHC
}

\author{
Michael Holmes and Brent D. Nelson \\ Department of Physics, Northeastern University, Boston, MA 02130, USA
}

(Dated: September 12, 2018)

\begin{abstract}
We consider the possibility that the recently reported events at the CDMS-II direct dark matter detection experiment are the result of coherent scattering of supersymmetric neutralinos. In such a scenario we argue that non-universal soft supersymmetry breaking gaugino masses are favored with a resulting lightest neutralino with significant Higgsino and wino components. We discuss the accompanying signals which must be seen at liquid-xenon direct detection experiments and indirect detection experiments if such a supersymmetric interpretation is to be maintained. We illustrate the possible consequences for early discovery channels at the LHC via a set of benchmark points designed to give rise to an observed event rate comparable to the reported CDMS-II data.
\end{abstract}

Introduction. It has been widely held for some time that one of the most attractive virtues of supersymmetry as a theory relevant at the electroweak scale is the possible presence of a stable, massive, neutral state in the superpartner spectrum capable of explaining the missing non-baryonic matter in the cosmos [1]. An excellent candidate for this dark matter particle is the lightest neutralino of supersymmetry [2, 3]. The diagrams which give rise to annihilation among relic neutralinos are among the same diagrams which determine the interaction rate of relic neutralinos in our local halo with terrestrial detectors. Therefore, supersymmetric models whose lightest supersymmetric particle (LSP) has the appropriate properties to account for the needed non-baryonic dark matter are often also models which could give rise to an observable signal in direct detection experiments. Establishing the existence of such a dark matter candidate via the observation of elastic scattering of the state off of target nuclei has been the pursuit of a variety of experiments for some period of time [4].

Recently, the CDMS-II collaboration has reported the observation of 2 nuclear recoil events consistent with the scattering of a weakly-interacting massive particle (WIMP) from germanium nuclei in a total exposure of $612 \mathrm{~kg}$-days [5]. The measured recoil energies for these two events were $12.3 \mathrm{keV}$ and $15.5 \mathrm{keV}$, near the low end of the recoil energies considered. In this energy range the CDMS collaboration estimates their signal efficiency to be no less than $25 \%$, reaching a maximum of $32 \%$ at $20 \mathrm{keV}$ recoil energies. The expected number of background events from mis-identified electron scattering events or neutron scattering events generated by radioactive decays and/or cosmic ray events was estimated to be $\Delta N=0.6 \pm 0.1$ (stat) prior to unblinding. This estimate was subsequently estimated to be $\Delta N=0.8 \pm 0.1$ (stat) \pm 0.2 (syst) after investigating the signal events in greater detail. It is therefore quite possible that these events are simply mis-identified background events. Taken as a signal they imply a coherent scattering cross-section for a WIMP in the local halo at roughly the few $\times 10^{-44} \mathrm{~cm}^{2}$ level.

If these events are interpreted as the scattering of a supersymmetric neutralino from the germanium components in the CDMS detector a number of very exciting corollaries may follow. The implications of these events has already been studied in a number of interesting model contexts [6, 7, 8, 9]. In this work we will outline some of the possible implications of the CDMS data on the nature of supersymmetric models generally. To illustrate these features we will consider a number of benchmark points - all of which could generate signals of the sort reported by the CDMS experiment. One of the most important conclusions is that some amount of non-universality in the gaugino soft supersymmetry-breaking masses may need to be present. We will discuss the crucial cross-checks and corroborating observations that must be seen in the next round of data-taking in both germanium- and xenon-based detectors for these conclusions to hold. We will also consider the prospects for indirect detection via the observation of the decay products from neutralino pair-annihilation at gamma-ray observatories and the neutrino observatory at IceCube. We will comment on the compatibility of any supersymmetric interpretation of the CDMS data with recent measurements of cosmic anti-matter fluxes from the PAMELA experiment and with thermal calculations of the relic density of stable neutralinos. Finally, we will briefly comment on what we may expect from the LHC at center-of-mass energies of 7, 10 and $14 \mathrm{TeV}$ for our candidate models.

\section{Direct Detection of Neutralino Recoils in Germanium.}

We begin with the methodology employed to translate the parameters of supersymmetric models into predictions of event rates at the CDMS-II in a given amount of exposure (fiducial volume $\times$ time of data collection). To compute the interaction rate of relic neutralinos with the nuclei of the target material one considers both spindependent (SD) and spin-independent (SI) interactions. For target nuclei with large atomic numbers the SI interaction, which is coherent across all of the nucleons in the nucleus, tends to dominate. The SI cross section $\sigma^{\mathrm{SI}}$ is computed in DarkSUSY [10] on an arbitrary nuclear target 
via

$$
\sigma_{\chi i}^{\mathrm{SI}}=\frac{\mu_{i \chi}^{2}}{\pi}\left|Z G_{s}^{p}+(A-Z) G_{s}^{n}\right|^{2},
$$

where $i$ labels the nuclear species in the detector with nuclear mass $M_{i}, \mu_{i \chi}$ is the reduced mass of the nucleus/neutralino system $\mu_{i \chi}=m_{\chi} M_{i} /\left(m_{\chi}+M_{i}\right)$, and $A$ and $Z$ are the target nucleus mass number and atomic number, respectively. The quantities $G_{s}^{p}$ and $G_{s}^{n}$ represent scalar four-fermion couplings of the neutralino to point-like protons and neutrons. They can be described schematically as

$$
G_{s}^{N}=\sum_{q=u, d, s, c, b, t}\langle N|\bar{q} q| N\rangle \times(\text { SUSY parameters }),
$$

where the quantity in parenthesis is calculable once the details of the supersymmetric model are specified. The initial nuclear matrix elements, however, are at present not calculable from first principles. Their values must be inferred from pion-nucleon scattering data. Depending on the methodology employed in this analysis, different values for this important set of parameters can be extracted - particularly for the case of the $\pi N \Sigma$ term [11, 12, 13]. The importance of the resulting uncertainty in this parameter on predictions for dark matter interaction cross-sections was recently considered in [14, 15, 16], where it was shown to be potentially quite large. We will return to this issue at the very end of this section. For what follows we will simply use the default values in DarkSUSY 5.0.4 for all nuclear matrix elements.

The differential rate of neutralino-nucleon scattering events (in units of events $/ \mathrm{kg} /$ day) can be computed from (11) according to

$$
\frac{d R}{d E}=\sum_{i} c_{i} \frac{\rho_{\chi} \sigma_{\chi i}\left|F_{i}\left(q_{i}\right)\right|^{2}}{2 m_{\chi} \mu_{i \chi}^{2}} \int_{v_{\min }}^{\infty} \frac{f(\vec{v}, t)}{v} d^{3} v,
$$

where we sum over all nuclear species present, with $c_{i}$ being the mass fraction of species $i$ in the detector. The quantity $f(\vec{v}, t) d^{3} v$ is the neutralino velocity distribution (presumed to be Maxwellian) with $v=|\vec{v}|$ the neutralino velocity relative to the detector [2]. Finally $\left|F_{i}\left(q_{i}\right)\right|^{2}$ is the nuclear form factor for species $i$, with $q_{i}=\sqrt{2 M_{i} E}$ being the momentum transfer for a nuclear recoil with energy $E$. For the purpose of this analysis we will use the output differential rates from DarkSUSY, calculated via (3), over a range of recoil energies relevant to the desired experiment. For the CDMS experiment the reported energy range considered was

$$
10 \mathrm{keV} \leq E_{\text {recoil }} \leq 100 \mathrm{keV} .
$$

We therefore perform a numerical integration of (3) by constructing an interpolating function for the differential rate sampled in $1 \mathrm{keV}$ intervals.
The sensitivity of the CDMS-II experiment, when translated into the size of the SI cross section $\sigma_{\chi p}^{\mathrm{SI}}$, is dependent on the mass of the LSP neutralino. For LSP masses in the range $100 \mathrm{GeV} \lesssim m_{\chi_{1}^{0}} \lesssim 300 \mathrm{GeV}$ a detectable event rate at CDMS-II implies a cross-section $\sigma_{\chi p}^{\mathrm{SI}} \gtrsim$ few $\times 10^{-44} \mathrm{~cm}^{2}$. If we assume the signal efficiency is $30 \%$ for the CDMS-II experiment we have an effective exposure of $184 \mathrm{~kg}$-days. For comparison to the data we note that a cross-section $\sigma_{\chi p}^{\mathrm{SI}}=10^{-44} \mathrm{~cm}^{2}$ for $m_{\chi_{1}^{0}} \simeq 150 \mathrm{GeV}$ implies approximately 0.5 expected events in this effective exposure.

A scalar-scalar cross-section this large is not generic in supersymmetric models, particularly those with universal gaugino masses that tend to give rise to an overwhelmingly bino-like LSP [16, 17]. Values of $\sigma_{\chi p}^{\mathrm{SI}}$ in the range for which CDMS-II is sensitive tend to involve LSPs with a sizable mixture of non-bino components, particularly Higgsino components [18, 19, 20, 21, 22, 23]. This is because the SI cross-section is dominated by t-channel Higgs exchange diagrams, the size of which are determined by the Higgsino component of the LSP. An observable signal at CDMS-II therefore also favors a relative light CP-even Higgs mass.

In Table \we present five benchmark points which exhibit event rates comparable to those recently reported by the CDMS-II collaboration, computed by integrating (3) over the range (4). We arrived at these points by considering a simple unified model with universal scalar soft mass $m_{0}$ and trilinear coupling $A_{0}$ at a highenergy scale, here taken to be the grand unified scale $\Lambda_{\mathrm{UV}}=2 \times 10^{16} \mathrm{GeV}$. We then introduced non-universal gaugino soft masses $M_{1}, M_{2}$ and $M_{3}$ via

$$
M_{i}=m_{1 / 2}\left(1+\delta_{i}\right)
$$

where $m_{1 / 2}$ represents the typical scale of the soft gaugino masses and we will consider only $\delta_{2}, \delta_{3} \neq 0$. This approach has been shown to give supersymmetric models compatible with all direct and indirect constraints, but with relatively large spin-independent cross-sections for the LSP neutralino [27, 28]. This approach is similar in spirit to the exploration conducted in [20, 21].

The gluino mass plays an important role in these models, albeit an indirect one. As is well known, the gluino soft mass $M_{3}$ at the high scale influences the eventual value of the $\mu$ parameter (as determined by solving the electroweak symmetry breaking conditions at the low energy scale) via renormalization group effects on the running of the Higgs scalar soft masses [29, 30, 31]. The consistent choice of $\delta_{3}<0$ is therefore preferred as this tends to decrease the value of the $\mu$ parameter and hence an increase in the Higgsino content of the eventual LSP.

The relevant information for connecting these models to the reported signal at CDMS-II is collected in Table III where we give the mass of the LSP neutralino, the spin-independent cross-section for elastic scattering on a single proton, and the number of events expected at 
TABLE I: Soft term parameters for selected points for comparison to reported CDMS signal. All soft mass parameters are given in units of $\mathrm{GeV}$ and all points were selected to have $\mu>0$.

\begin{tabular}{|c|c|c|c|c|c|}
\hline \hline Point & $\mathrm{A}$ & $\mathrm{B}$ & $\mathrm{C}$ & $\mathrm{D}$ & $\mathrm{E}$ \\
\hline \hline$m_{0}$ & 750 & 500 & 350 & 350 & 350 \\
$m_{1 / 2}$ & 750 & 500 & 575 & 575 & 575 \\
$A_{0}$ & 370 & 270 & 150 & 150 & 150 \\
$\tan \beta$ & 25 & 15 & 30 & 30 & 30 \\
\hline$\delta_{2}$ & 0.65 & 0.62 & -0.6 & 0.82 & -0.47 \\
$\delta_{3}$ & -0.35 & -0.3 & -0.3 & -0.35 & -0.3 \\
\hline \hline
\end{tabular}

TABLE II: Properties of the LSP and resulting CDMS-II recoil rate for examples in Table [ Masses and LSP properties were computed using SuSpect 2.41, while scattering crosssections and the expected number of events were computed using default values in DarkSUSY 5.0.4. These numbers carry uncertainties associated with certain nuclear matrix elements, as mentioned in the text. Event rates on germanium nuclei assume an effective exposure of $183.6 \mathrm{~kg}$-days.

\begin{tabular}{|c|c|c|c|c|c|}
\hline \hline Point & $\mathrm{A}$ & $\mathrm{B}$ & $\mathrm{C}$ & $\mathrm{D}$ & $\mathrm{E}$ \\
\hline \hline$m_{\chi_{1}^{0}}(\mathrm{GeV})$ & 138 & 190 & 175 & 112 & 230 \\
\hline $\mathrm{B} \%$ & $3.0 \%$ & $70.2 \%$ & $0.3 \%$ & $5.4 \%$ & $40.9 \%$ \\
$\mathrm{~W} \%$ & $0.4 \%$ & $0.4 \%$ & $95.8 \%$ & $0.5 \%$ & $53.0 \%$ \\
$\mathrm{H} \%$ & $96.6 \%$ & $29.4 \%$ & $3.9 \%$ & $94.1 \%$ & $6.1 \%$ \\
\hline$\sigma_{\chi p}^{\mathrm{SI}} \times 10^{45}\left(\mathrm{~cm}^{2}\right)$ & 11.9 & 44.4 & 41.3 & 35.3 & 74.8 \\
$N_{\mathrm{Ge}}(184 \mathrm{~kg}$-days $)$ & 0.51 & 1.36 & 1.30 & 1.65 & 1.90 \\
\hline \hline
\end{tabular}

CDMS-II over the energy range in (4) with an effective exposure of $0.3 \times 612=183.6 \mathrm{~kg}$-days. We have deliberately chosen examples with a variety of neutralino mass matrices, as evidenced by the eigenvector of the lowest mass state. This LSP wavefunction composition is indicated by the entries $\mathrm{B} \%, \mathrm{~W} \%$ and $\mathrm{H} \%$ which give the percentage of bino eigenstate, wino eignesate and Higgsino eigenstate, respectively. These quantities were computed using SuSpect 2.4132 and DarkSUSY.

Given the low statistics in the reported CDMS data we believe that any one of these models could conceivably be giving rise to the observed recoils. As mentioned above, these interaction rates are sensitive to still uncertain input numbers associated with certain nuclear matrix elements. For example, the package MicrOMEGAs [34] uses a different default value for the $\pi N \Sigma$-term and consequently predicts an event rate on germanium roughly twice as large as the values give in Table [I] We have therefore chosen examples with $0.5 \leq N_{\mathrm{Ge}} \leq 2.0$ to be conservative in our estimates. It should also be noted that recent calculations of the matrix element $\langle n|s \bar{s}| n\rangle$ 35] may imply a much lower effective value for $\sigma_{\chi p}^{\text {SI }}$, implying a more challenging environment for interpreting the CDMS-II events as
TABLE III: Predictions of the benchmark models in Table I for other dark matter search experiments and the thermal relic density of neutralinos. All calculations were performed using DarkSUSY 5.0.4.

\begin{tabular}{|c|c|c|c|c|c|}
\hline \hline Point & $\mathrm{A}$ & $\mathrm{B}$ & $\mathrm{C}$ & $\mathrm{D}$ & $\mathrm{E}$ \\
\hline \hline$R_{\mathrm{Xe}}\left(\mathrm{kg}^{-1}-\mathrm{yr}^{-1}\right)$ & 1.32 & 3.64 & 3.66 & 4.63 & 5.15 \\
$N_{\text {Xe }}($ Xenon10) & 1.14 & 3.16 & 3.17 & 4.01 & 4.47 \\
$N_{\mathrm{Xe}}(\mathrm{Xenon} 100)$ & 17.4 & 47.9 & 48.1 & 60.9 & 67.7 \\
\hline$\left(\Phi_{\text {int }} / \bar{J}\right) \times 10^{15}\left(\mathrm{~cm}^{-2} \mathrm{~s}^{-1}\right)$ & 196.8 & 9.27 & 1020.4 & 243.8 & 164.1 \\
$\Phi_{\gamma \gamma /} \Phi_{\gamma Z}$ & 0.52 & 0.20 & 0.33 & 0.57 & 0.31 \\
\hline$\Phi_{\mu}\left(\mathrm{km}^{-2} \mathrm{yr}^{-1}\right)$ & 114.0 & 171.3 & 63.4 & 130.7 & 123.5 \\
\hline$\langle\sigma v\rangle_{W W} \times 10^{25}\left(\mathrm{~cm}^{2}\right)$ & 1.9 & 0.1 & 23.8 & 2.0 & 4.5 \\
\hline$\left.\Omega \mathrm{h}^{2}\right|_{\text {therm }}$ & 0.005 & 0.112 & 0.001 & 0.006 & 0.003 \\
\hline \hline \hline
\end{tabular}

neutralino scatters [36]. Of course accumulating more statistics may allow for a crude measurement of the recoil energy spectrum and thus shed more light on the nature of the scattering particle.

\section{Implications for Other Dark Matter Search Experi-} ments.

As we saw in the previous section, a variety of LSP configurations can give rise to the signal seen at CDMS-II. Given the low statistics in the reported data it is not altogether clear whether the events seen truly indicate the presence of new physics. Additional data collection is, of course, imperative to a firm determination that we are indeed seeing evidence of dark matter elastic scatters with an effective cross-section of the order $\sigma \simeq 10^{-44} \mathrm{~cm}^{2}$.

One crucial cross-check will be the next round of results reported at xenon-based detectors. The spinindependent interaction rates of neutralinos on germanium and on xenon are highly correlated. Interestingly, the implied rate for neutralinos scattering on xenon inferred from the CDMS-II results implies something on the order of one to five events in an exposure of $316.4 \mathrm{~kg}$ days at Xenon10. Ten events were in fact observed in the region where the signal was expected, but this was consistent with their background estimation [37]. A confirming signal from a liquid-xenon based detector in the near future is therefore imperative for the supersymmetric interpretation of the CDMS events.

To compute the rate of elastic scattering on xenonbased targets we modify the procedure of the previous section and use an integration range for (3) that better reflects the sensitivities of the Xenon10 apparatus:

$$
5 \mathrm{keV} \leq E_{\text {recoil }} \leq 25 \mathrm{keV} .
$$

The event rates for our benchmark points in units of recoils $/ \mathrm{kg} /$ year are given in the first entry of Table III. In the second entry we give the expected number of signal events in $316.4 \mathrm{~kg}$-days at Xenon10. These points are clearly in a region where the liquid-xenon experiments 
should be sensitive to a signal. In the third entry we give the expected event rate at the Xenon100 upgrade assuming an exposure of $80 \mathrm{~kg} \times 60$ days $=13.15 \mathrm{~kg}$-years. Assuming adequate rejection of background events all of these candidate models should give clear dark matter signals in the earliest stages of running at Xenon100.

Observation of a clear signal above background in liquid xenon-based detectors would (a) provide some assurance that the effect seen at CDMS-II is truly the result of dark matter scattering, and (b) give some further support to the conjecture that this involves elastic scattering of supersymmetric neutralinos. Even within the supersymmetric framework, however, it is unlikely that such an observation alone can distinguish between the various models represented by the benchmarks in Table [1 Several indirect detection experiments, however, may be sensitive to the differences in the LSP wavefunction that distinguish these models.

The Fermi-GLAST satellite is currently surveying the flux of gamma ray photons from the location of the galactic center. If some component of this flux comes from the annihilation of LSP neutralinos it may be possible to observe an increase in the flux over estimated astrophysical backgrounds. The calculation of the flux depends on the microscopic physics of the neutralino and its interactions, but also very strongly on the macroscopic physics of the halo profile assumed for the galaxy. The latter is conveniently summarized by a single parameter $\bar{J}(\Delta \Omega)$ where $\Delta \Omega$ represents the solid angle resolution of the observatory. We consider the commonly adopted profile of Navarro, Frank and White (NFW) 38] which gives a value of $\bar{J}(\Delta \Omega)=1.2644 \times 10^{4}$ and assume an angular resolution of $\Delta \Omega=10^{-5}$ sr. The value of the estimated gamma ray flux for photons in the energy range $1 \mathrm{GeV} \leq E_{\gamma} \leq 200 \mathrm{GeV}$ is given in Table III. The expected flux is generally quite large, suggesting that a signal should be expected above background at Fermi/GLAST in the near future. But we emphasize that these estimates can vary over many orders of magnitude if different assumptions about the halo profile are employed.

In addition to the diffuse gamma rays coming from neutralino annihilations one also expects monochromatic signals from loop-induced diagrams in which two neutralinos annihilate into a pair of photons ( $\gamma \gamma$ line) or into a photon and a Z-boson ( $\gamma Z$ line) 39, 40, 41]. It has been noted that the ratio of the observed gamma ray fluxes from these two processes is correlated with the wave-function composition of the LSP [42]. Furthermore, taking the ratio of the fluxes eliminates much of the halomodel dependence from the analysis of the signal. The ratio of the fluxes $\Phi_{\gamma \gamma} / \Phi_{\gamma Z}$ is given in Table III for our various benchmark points. They differ by as much as a factor of two. Observing these ratios, however, at atmospheric Chernekov telescopes will require a favorable halo model profile and excellent energy resolution on the measurement of these high-energy photons.

More promising signals may come from the search for neutrinos arising from the annihilation of LSP neutralinos in a location closer to the earth, such as the center of the sun, where issues of the galactic halo do not come into play. Again we expect relatively large differences in the flux of neutrinos between wino-like and Higgsino-like LSPs. Some fraction of the neutrinos can eventually exit the sun and be detected in experiments such as IceCube [43] via conversion of muon neutrinos into muons. To calculate this rate we integrate the differential flux of conversion muons from solar-born as well as earth-born neutrinos over the energy range $50 \mathrm{GeV} \leq E_{\mu} \leq 300 \mathrm{GeV}$, assuming an angular resolution of 3 degrees. The nominal target area for IceCube is $1 \mathrm{~km}^{2}$, but the effective area for detection of neutrinos via muon conversion is smaller and can be cast as a function of the muon energy [44, 45, 46]. Previous observations based on a limited set of 22 strings of photomultiplier tubes put a limit on the size of the muon flux at approximately $300-400$ muons $/ \mathrm{km}^{2} /$ year. The fluxes for these benchmark models all fall well below these limits, as shown in Table III though well within reach of the next data run at IceCube. Even with an exposure of only $0.2 \mathrm{~km}^{2}$-years all of these models will produce at least 10 signal events at IceCube.

Finally, we mention the implications for the observed excess in positrons in the energy range $10-100 \mathrm{GeV}$ recently measured by the PAMELA experiment [47]. It is by now well established that standard MSSM models can fit the signal from PAMELA only with some difficulty. For models with a significant wino or Higgsino content, the observed rate implies a thermally-averaged annihilation cross-section in to $W^{+} W^{-}$final states on the order of $\langle\sigma v\rangle_{W W} \sim 10^{-24} \mathrm{~cm}^{3} / \mathrm{s}$ [48, 49]. Such rates can be achieved for some of the models in Table III though not for all. ${ }^{1}$ Models which achieve this high annihilation rate tend to also be cases in which neutralinos annihilate very effectively in the early universe, resulting in a diminished thermal relic density. We chose one model (Point B) which gives a thermal relic density, as computed using DarkSUSY, which fits the measurement as inferred from the WMAP three-year [51] and five-year data [52] which we take to be

$$
0.0855 \leq \Omega_{\chi} \mathrm{h}^{2} \leq 0.1189
$$

at the $2 \sigma$ level. For the other cases it will clearly be necessary to invoke non-thermal production [53, 54, 55, 56] to achieve the appropriate relic density.

\section{Implications for Observation of SUSY at the LHC.}

\footnotetext{
${ }^{1}$ See also the benchmark cases given in [50]
} 
TABLE IV: Relevant SUSY mass spectra and total production cross sections at the LHC, for the benchmark models in Table [1. All masses are in GeV.

\begin{tabular}{|c|r|r|r|r|r|}
\hline \hline Point & A & B & C & D & E \\
\hline \hline$m_{\chi_{1}^{0}}$ & 138 & 190 & 175 & 112 & 230 \\
$m_{\chi_{2}^{0}}$ & 152 & 254 & 235 & 130 & 239 \\
$m_{\chi_{3}^{0}}$ & 326 & 261 & 505 & 252 & 504 \\
$m_{\chi_{4}^{0}}$ & 1008 & 663 & 513 & 846 & 515 \\
$m_{\chi_{1}^{ \pm}}$ & 146 & 243 & 175 & 123 & 234 \\
$m_{\chi_{2}^{ \pm}}$ & 1008 & 663 & 514 & 846 & 515 \\
\hline$m_{\tilde{g}}$ & 1156 & 847 & 952 & 890 & 951 \\
$m_{\tilde{t}_{1}}$ & 826 & 607 & 719 & 544 & 709 \\
$m_{\tilde{t}_{2}}$ & 1284 & 925 & 862 & 964 & 865 \\
$m_{\tilde{b}_{1}}$ & 1155 & 853 & 809 & 766 & 812 \\
$m_{\tilde{b}_{2}}$ & 1271 & 903 & 874 & 943 & 871 \\
\hline$m_{\tilde{\tau}_{1}}$ & 740 & 520 & 344 & 338 & 352 \\
$m_{\tilde{\tau}_{2}}$ & 1079 & 724 & 414 & 752 & 424 \\
$m_{h}$ & 115 & 112 & 113 & 114 & 113 \\
\hline$\sigma_{\mathrm{STSY}}^{7 \mathrm{TeV}}(\mathrm{pb})$ & 1.3 & 0.3 & 1.2 & 2.7 & 0.4 \\
$\sigma_{\mathrm{SUSY}}^{10 \mathrm{TeV}}(\mathrm{pb})$ & 2.3 & 1.2 & 2.5 & 5.1 & 1.3 \\
$\sigma_{\mathrm{SUSY}}^{14 \mathrm{TeV}}(\mathrm{pb})$ & 4.0 & 4.1 & 5.7 & 10.0 & 3.7 \\
\hline \hline \hline
\end{tabular}

A common feature of the models listed in Table $\Pi$ is a general compression of the gaugino mass spectrum relative to unified models such as mSUGRA. This is particularly true of the mass gap $\Delta^{ \pm} \equiv m_{\chi_{1}^{ \pm}}-m_{\chi_{1}^{0}}$ which is crucial to many of the standard supersymmetric search strategies at the LHC, as well as having a large impact on the triggering efficiencies for supersymmetric events. We give certain key physical masses for the models of Table \ in Table IV in units of GeV. All masses are computed from the high-scale boundary conditions after renormalization group evolution using SuSpect 2.41.

To analyze the signatures of these models at the LHC we generated events using PYTHIA followed by a detector simulation using PGS4 [57]. Sparticle decays are calculated using SUSY-HIT [33]. Data sets representing $1 \mathrm{fb}^{-1}$ were generated for center of mass energies $\sqrt{s}=10 \mathrm{TeV}$ and $\sqrt{s}=14 \mathrm{TeV}$ for each model. In addition we considered a suitably-weighted sample of $1 \mathrm{fb}^{-1}$ Standard Model background events, consisting of DrellYan, QCD dijet, $t \bar{t}, b \bar{b}, W / Z+$ jets and diboson production at each $\sqrt{s}$ value. Events were analyzed using level one (L1) triggers in PGS4, designed to mimic the CMS trigger tables [58]. Object-level post-trigger cuts were also imposed. We require all photons, electrons, muons and taus to have transverse momentum $p_{T} \geq 10 \mathrm{GeV}$ and $|\eta|<2.4$ and we require hadronic jets to satisfy $|\eta|<3$.

The last entries of Table IV gives the total SUSY production cross-section at the LHC for center of mass energies of 7,10 and $14 \mathrm{TeV}$ for our benchmark points.
TABLE V: Number of signal events for standard discovery channels at the LHC for $\sqrt{s}=10 \mathrm{TeV}$ for the benchmark models of Table $\square$

\begin{tabular}{|c|c|r|r|r|r|}
\hline \multicolumn{7}{c}{ Numbers of Events } \\
\hline Point & $\mathrm{A}$ & $\mathrm{B}$ & $\mathrm{C}$ & $\mathrm{D}$ & $\mathrm{E}$ \\
\hline \hline Multijets & 16 & 68 & 72 & 114 & 57 \\
$1 \ell+$ jets & 17 & 78 & 61 & 70 & 19 \\
OS $2 \ell+$ jets & 0 & 14 & 2 & 12 & 1 \\
$\mathrm{SS} 2 \ell+$ jets & 0 & 1 & 2 & 4 & 0 \\
$3 \ell+$ jets & 0 & 4 & 0 & 4 & 0 \\
\hline \multicolumn{7}{c|}{ Significance $S / \sqrt{B}$} & & \\
\hline \hline Point & $\mathrm{A}$ & $\mathrm{B}$ & $\mathrm{C}$ & $\mathrm{D}$ & $\mathrm{E}$ \\
\hline $1 \ell+$ jets & 1.1 & 5.2 & 4.1 & 4.7 & 1.7 \\
OS $2 \ell+$ jets & $\mathrm{NA}$ & 4.4 & 0.6 & 3.8 & 0.3 \\
$\mathrm{SS} 2 \ell+$ jets & $\mathrm{NA}$ & 1.0 & 2.0 & 4.0 & $\mathrm{NA}$ \\
$3 \ell+$ jets & $\mathrm{NA}$ & 2.9 & $\mathrm{NA}$ & 2.9 & $\mathrm{NA}$ \\
\hline \hline
\end{tabular}

Given the relatively low cross-sections and reduction in leptonic signatures (as we describe below) it is highly unlikely that these models will give significant event rates above the Standard Model background in $500 \mathrm{pb}^{-1}$ or less integrated luminosity for $\sqrt{s}=7 \mathrm{TeV}$. However, the prospects brighten considerably at higher energies, particularly if one assumes that measurements of jet $p_{T}$ and $E_{T}$ are reasonably reliable.

We begin with standard SUSY discovery modes [59], slightly modified to maximize the signal significance for these models. These five signatures are collected in Table $\mathrm{V}$ for $1 \mathrm{fb}^{-1}$ of integrated luminosity at $\sqrt{s}=10 \mathrm{TeV}$. In all cases we require transverse sphericity $S_{T} \geq 0.2$ and at least $250 \mathrm{GeV}$ of $E_{T}$ except for the trilepton signature, where only $E_{T} \geq 200 \mathrm{GeV}$ is required. The multijet channel includes a veto on isolated leptons and requires at least four jets with the transverse momenta of the four leading jets satisfying $p_{T} \geq(200,150,50,50) \mathrm{GeV}$, respectively. For the leptonic signatures we include only $e^{ \pm}$and $\mu^{ \pm}$final states and demand at least two jets with the leading jets satisfying $p_{T} \geq(100,50) \mathrm{GeV}$, respectively. We also display results in Table VI for $1 \mathrm{fb}^{-1}$ of integrated luminosity at $\sqrt{s}=14 \mathrm{TeV}$.

The multijet channel is promising already at $1 \mathrm{fb}^{-1}$ at $\sqrt{s}=10 \mathrm{TeV}$ for all models with the exception of point $\mathrm{A}$, which has the largest gluino mass. Points which give a mass difference $\Delta^{ \pm} \gtrsim 10 \mathrm{GeV}$ are capable of giving hard enough leptons to produce a reasonable signal significance in the one-, and even two-lepton channels. The compressed spectrum tends to result in fewer leptonic events due to the inability to trigger effectively on leptons when their transverse momentum drops much below $10 \mathrm{GeV}$. The reduction in leptonic signal is likely to be a generic property of supersymmetric models that 
TABLE VI: Number of signal events for standard discovery channels at the LHC for $\sqrt{s}=14 \mathrm{TeV}$ for the benchmark models of Table @

\begin{tabular}{|c|c|r|r|r|r|}
\hline \multicolumn{7}{c}{ Numbers of Events } \\
\hline Point & $\mathrm{A}$ & $\mathrm{B}$ & $\mathrm{C}$ & $\mathrm{D}$ & $\mathrm{E}$ \\
\hline \hline Multijets & 99 & 321 & 402 & 436 & 298 \\
$1 \ell+$ jets & 62 & 336 & 202 & 310 & 111 \\
OS $2 \ell+$ jets & 8 & 45 & 12 & 45 & 7 \\
SS $2 \ell+$ jets & 2 & 19 & 6 & 16 & 3 \\
$3 \ell+$ jets & 3 & 8 & 4 & 6 & 1 \\
\hline \multicolumn{7}{c|}{ Point } & $\mathrm{A}$ & $\mathrm{B}$ & $\mathrm{C}$ & $\mathrm{D}$ & $\mathrm{E}$ \\
\hline \hline Multijets & 6.6 & 21.4 & 26.9 & 29.1 & 19.9 \\
$1 \ell+$ jets & 2.5 & 13.6 & 8.2 & 12.5 & 4.5 \\
OS $2 \ell+$ jets & 1.3 & 7.4 & 2.0 & 7.4 & 1.2 \\
SS $2 \ell+$ jets & 0.8 & 7.2 & 2.3 & 6.0 & 1.1 \\
$3 \ell+$ jets & 1.2 & 3.3 & 1.6 & 2.5 & 0.4 \\
\hline \hline
\end{tabular}

give rise to large event rates at CDMS-II. On the other hand, all models should give rise to detectable signals at $\sqrt{s}=14 \mathrm{TeV}$ in multiple channels. We also note that all should have at least three states which can be produced and observed at a $\sqrt{s}=500 \mathrm{GeV} e^{+} e^{-}$linear collider. This is also likely to be a generic property of models which fit the CDMS-II signal well.

\section{Conclusions.}

If the events observed in the recent CDMS-II fivetower results are interpreted as scattering of supersymmetric neutralinos, we may begin to construct a picture of what to expect at future experiments. It is highly likely, though by no means necessary, that the soft supersymmetry breaking gaugino masses of the MSSM are non-universal when computed at some high-energy input scale. The CDMS-II results would favor a suppression of the gluino mass relative to the prediction of models such as mSUGRA. They may also imply a relatively compressed spectrum in the gaugino sector and possible diminishment of lepton-based discovery channels at the LHC. If a predominantly wino-like or Higgsino-like neutralino is the LSP we may be forced to consider scenarios which give rise to non-thermal production of relic neutralinos, such as those motivated from string theory [60]. In all scenarios which can give rise to $\mathcal{O}(1)$ events in the reported effective exposure at CDMS-II we should expect clear corroborating signals at the next round of liquid-xenon based direct detection experiments as well as clear signals at indirect detection experiments, most notably the neutrino-induced muons at IceCube.

\section{Acknowedgements.}

This work was supported by National Science Foundation Grant PHY-0653587.
[1] H. Goldberg, Phys. Rev. Lett. 50, 1419 (1983) [Erratumibid. 103, 099905 (2009)].

[2] G. Jungman, M. Kamionkowski and K. Griest, Phys. Rept. 267, 195 (1996).

[3] G. Bertone, D. Hooper and J. Silk, Phys. Rept. 405, 279 (2005).

[4] R. J. Gaitskell, Ann. Rev. Nucl. Part. Sci. 54, 315 (2004).

[5] Z. Ahmed et al. [The CDMS Collaboration], "Results from the Final Exposure of the CDMS II Experiment," arXiv:0912.3592 [astro-ph.CO].

[6] M. Kadastik, K. Kannike, A. Racioppi and M. Raidal, arXiv:0912.3797 [hep-ph].

[7] A. Bottino, F. Donato, N. Fornengo and S. Scopel, arXiv:0912.4025 [hep-ph].

[8] D. Feldman, Z. Liu and P. Nath, arXiv:0912.4217 [hep$\mathrm{ph}]$.

[9] M. Ibe and T. T. Yanagida, arXiv:0912.4221 [hep-ph].

[10] P. Gondolo, J. Edsjo, P. Ullio, L. Bergstrom, M. Schelke and E. A. Baltz, JCAP 0407, 008 (2004).

[11] A. Bottino, F. Donato, N. Fornengo and S. Scopel, Astropart. Phys. 13, 215 (2000).

[12] A. Bottino, F. Donato, N. Fornengo and S. Scopel, Astropart. Phys. 18, 205 (2002).

[13] M. M. Pavan, I. I. Strakovsky, R. L. Workman and R. A. Arndt, PiN Newslett. 16, 110 (2002).

[14] J. Ellis, K. A. Olive and C. Savage, Phys. Rev. D 77, 065026 (2008).

[15] A. Bottino, F. Donato, N. Fornengo and S. Scopel, Phys. Rev. D 78, 083520 (2008).

[16] J. R. Ellis, K. A. Olive, Y. Santoso and V. C. Spanos, Phys. Rev. D 71, 095007 (2005).

[17] J. R. Ellis, J. L. Feng, A. Ferstl, K. T. Matchev and K. A. Olive, Eur. Phys. J. C 24, 311 (2002).

[18] A. Birkedal-Hansen and B. D. Nelson, Phys. Rev. D 67, 095006 (2003).

[19] U. Chattopadhyay and D. P. Roy, Phys. Rev. D 68, 033010 (2003).

[20] H. Baer, A. Mustafayev, E. K. Park, S. Profumo and X. Tata, JHEP 0604, 041 (2006).

[21] H. Baer, A. Mustafayev, H. Summy and X. Tata, JHEP 0710, 088 (2007).

[22] K. J. Bae, R. Dermisek, H. D. Kim and I. W. Kim, JCAP 0708, 014 (2007).

[23] U. Chattopadhyay, D. Das and D. P. Roy, Phys. Rev. D 79, 095013 (2009).

[24] H. Baer, A. Mustafayev, S. Profumo, A. Belyaev and X. Tata, Phys. Rev. D 71, 095008 (2005).

[25] H. Baer, A. Mustafayev, S. Profumo, A. Belyaev and X. Tata, JHEP 0507, 065 (2005).

[26] J. Ellis, K. A. Olive and P. Sandick, New J. Phys. 11, 105015 (2009).

[27] A. Corsetti and P. Nath, Phys. Rev. D 64, 125010 (2001).

[28] A. Birkedal-Hansen and B. D. Nelson, Phys. Rev. D 64, 015008 (2001).

[29] G. L. Kane and S. F. King, Phys. Lett. B 451, 113 (1999).

[30] G. L. Kane, J. D. Lykken, B. D. Nelson and L. T. Wang, Phys. Lett. B 551, 146 (2003).

[31] D. Horton and G. G. Ross, arXiv:0908.0857 [hep-ph].

[32] A. Djouadi, J. L. Kneur and G. Moultaka, Comput. Phys. Commun. 176, 426 (2007).

[33] A. Djouadi, M. M. Muhlleitner and M. Spira, Acta Phys. 
Polon. B 38, 635 (2007).

[34] G. Belanger, F. Boudjema, A. Pukhov and A. Semenov, Comput. Phys. Commun. 180, 747 (2009).

[35] D. Toussaint and W. Freeman [MILC Collaboration], Phys. Rev. Lett. 103, 122002 (2009).

[36] J. Giedt, A. W. Thomas and R. D. Young, "Dark matter, the CMSSM and lattice QCD," arXiv:0907.4177 [hep-ph].

[37] J. Angle et al. [XENON Collaboration], Phys. Rev. Lett. 100, 021303 (2008).

[38] J. F. Navarro, C. S. Frenk and S. D. M. White, Astrophys. J. 462, 563 (1996);

J. F. Navarro, C. S. Frenk and S. D. M. White, Astrophys. J. 490, 493 (1997).

[39] L. Bergstrom and P. Ullio, Nucl. Phys. B 504, 27 (1997).

[40] Z. Bern, P. Gondolo and M. Perelstein, Phys. Lett. B 411, 86 (1997).

[41] P. Ullio and L. Bergstrom, Phys. Rev. D 57, 1962 (1998).

[42] P. Ullio, JHEP 0106, 053 (2001).

[43] F. Halzen, Eur. Phys. J. C 46, 669 (2006).

[44] M. C. Gonzalez-Garcia, F. Halzen and M. Maltoni, Phys. Rev. D 71, 093010 (2005) arXiv:hep-ph/0502223.

[45] F. Halzen and D. Hooper, Phys. Rev. D 73, 123507 (2006).

[46] V. Barger, W. Y. Keung, G. Shaughnessy and A. Tregre, Phys. Rev. D 76, 095008 (2007).

[47] O. Adriani et al. [PAMELA Collaboration], Nature 458, 607 (2009).
[48] M. Cirelli, M. Kadastik, M. Raidal and A. Strumia, Nucl. Phys. B 813, 1 (2009).

[49] P. Grajek, G. Kane, D. Phalen, A. Pierce and S. Watson, Phys. Rev. D 79, 043506 (2009).

[50] D. Feldman, Z. Liu, P. Nath and B. D. Nelson, Phys. Rev. D 80, 075001 (2009).

[51] D. N. Spergel et al. [WMAP Collaboration], Astrophys. J. Suppl. 170, 377 (2007).

[52] E. Komatsu et al. [WMAP Collaboration], Astrophys. J. Suppl. 180, 330 (2009).

[53] T. Moroi and L. Randall, Nucl. Phys. B 570, 455 (2000).

[54] M. Nagai and K. Nakayama, Phys. Rev. D 76, 123501 (2007).

[55] M. Nagai and K. Nakayama, Phys. Rev. D 78, 063540 (2008).

[56] G. B. Gelmini and P. Gondolo, Phys. Rev. D 74, 023510 (2006).

[57] P. Skands et al., JHEP 0407, 036 (2004); PGS-4, J. Conway et. al.

[58] G. L. Bayatian et al. [CMS Collaboration], J. Phys. G 34, 995 (2007).

[59] H. Baer, C. h. Chen, F. Paige and X. Tata, Phys. Rev. D 52, 2746 (1995); H. Baer, C. h. Chen, F. Paige and X. Tata, Phys. Rev. D 53, 6241 (1996).

[60] S. Watson, arXiv:0912.3003 [hep-th]. 